Documentation et bibliothèques

DOCUMENTATION BIBLIOTHEQUES

\title{
De la carte à la cartothéconomie : l'émergence d'un secteur documentaire qui découvre son identité
}

\section{Yves Tessier}

Volume 25, numéro 2, juin 1979

URI : https://id.erudit.org/iderudit/1054358ar

DOI : https://doi.org/10.7202/1054358ar

Aller au sommaire du numéro

Éditeur(s)

Association pour l'avancement des sciences et des techniques de la documentation (ASTED)

ISSN

0315-2340 (imprimé)

2291-8949 (numérique)

Découvrir la revue

Citer cet article

Tessier, Y. (1979). De la carte à la cartothéconomie : l'émergence d'un secteur documentaire qui découvre son identité. Documentation et bibliothèques, 25(2), 71-80. https://doi.org/10.7202/1054358ar
Résumé de l'article

La documentation cartographique communique l'information géographique en respectant son caractère spatial et interrelationnel, deux aspects intimement liés qui définissent l'essence même de l'esprit géographique. Cette spécificité informationnelle aide à établir le rôle que peut jouer ce support dans une bibliothèque ou un centre d'information. Son organisation générale se modèle sur celle de la bibliothéconomie spécialisée et a donné naissance à un secteur professionnel qui a développé son identité propre, la cartothéconomie.
Tous droits réservés (C) Association pour l'avancement des sciences et des techniques de la documentation (ASTED), 1979
Ce document est protégé par la loi sur le droit d'auteur. L’utilisation des services d'Érudit (y compris la reproduction) est assujettie à sa politique d'utilisation que vous pouvez consulter en ligne.

https://apropos.erudit.org/fr/usagers/politique-dutilisation/ 


\title{
De la carte à la cartothéconomie: l'émergence d'un secteur documentaire qui découvre son identité
}

\author{
Yves Tessier \\ Directeur \\ Secteur de l'exploitation et du développement \\ Bibliothèque de l'Université Laval \\ Québec
}

La documentation cartographique communique l'information géographique en respectant son caractère spatial et interrelationnel, deux aspects intimement liés qui définissent l'essence même de l'esprit géographique. Cette spécificité informationnelle aide à établir le rôle que peut jouer ce support dans une bibliothèque ou un centre d'information. Son organisation générale se modèle sur celle de la bibliothéconomie spécialisée et a donné naissance à un secteur professionnel qui a développé son identité propre, la cartothéconomie.

Cartographic materials conveys geographic information by respecting its spatial and relation-oriented characteristics. These aspects are typical of the geographic approach. This exclusiveness among information medias brings out the role of cartographic materials in libraries and information centers. Organizational patterns of this medium follow those of special librarianship and have evolved towards an area with an identity of its own, map librarianship.

La documentación cartográfica comunica la información geográfica y respeta su carácter espacial e interrelacional, dos aspectos que definen la esencia del espiritu geográfico. Esta especifidad de la información contribuye a establecer el papel que puede tener la documentación cartográfica en una biblioteca o un centro de información. Su organización general sigue el modelo de la biblioteconomía especializada y ha creado un sector profesional que ha desarrollado su identidad propia: la cartoteconomía.

Le milieu documentaire a mis du temps à recevoir les lettres de créance de la documentation cartographique comme support de l'information. Lors du congrès de l'automne 1978 de l'ASTED, on parlait encore de documents «inusités» pour désigner les cartes géographiques. Bien sûr l'inusité, c'est très relatif... comme le fait que le mot cartothèque n'apparaisse pas

N.D.L.R. L'auteur a été chef de la cartothèque de la bibliothèque de l'Université Laval de 1964 à 1979. encore au Dictionnaire alphabétique et analogique de la langue française (Petit Robert).

Pourtant le rattrapage a été extrêmement rapide. La distribution massive de surplus de guerre cartographiques aux États-Unis, après la deuxième guerre mondiale, a permis la création de collections de cartes qui ne cessent de se développer depuis. Les progrès de l'enseignement de la géographie en milieu universitaire et 
l'éveil, même s'il a été tardif, de la bibliothéconomie à ce nouveau support documentaire ont favorisé la maturation de la moisson, clairsemée certes mais prometteuse, de collections de cartes organisées fonctionnellement sur le modèle des bibliothèques spécialisées. Ce nouveau secteur de l'information scientifique est décrit par analogie en termes de cartothèque (map library), de cartothécaire (map librarian) et de cartothéconomie (map librarianship).

On peut même affirmer avec fierté que nous, les spécialistes des cartes géographiques, avons «notre» ISBD, «notre» format MARC et «nos» AACR 2!

Le présent article a pour but d'esquisser les principaux traits spécifiques de la documentation cartographique comme support de l'information scientifique et de fournir des indications utiles concernant l'organisation et l'exploitation de ce médium dans un centre documentaire.

\section{La carte géographique: un médium scriptovisuel de communication}

Parler de cartes géographiques, c'est évoquer les cartes du relief, les cartes des divisions politiques, les cartes routières, c'est-à-dire les cartes axées sur la localisation de divers phénomènes considérés isolément. La cartographie s'est considérablement renouvelée ces dernières années pour refléter davantage l'approche multidisciplinaire et à tendance synthétisante (écologique) de la géographie et pour tenir compte également des progrès du langage graphique. En conséquence, la production cartographique s'est orientée davantage dans le sens de la création d'un médium scriptovisuel de communication destiné à faciliter la compréhension globale des phénomènes représentés (plutôt que leur simple représentation). II est utile de connaître les caractéristiques de cette évolution récente dans le but de bien cerner le rôle spécifique du document cartographique parmi les supports de l'information scientifique.

\section{La spécificité informationnelle du document cartographique}

La carte jouit d'une double spécificité informationnelle. La première touche au caractère spatial (la répartition dans l'espace) dans la représentation de l'information sur le modèle réduit (à l'échelle) qu'est le document cartographique. Un traité, si élaboré soit-il, sur la géologie d'une région donnée ne parviendra jamais à remplacer la carte géologique de la même région. Comment décrire de façon efficace la nature des diverses couches géologiques et leur répartition sur le terrain autrement que par une carte?

L'autre spécificité informationnelle tient à la figuration des interre/ations qui existent entre les phénomènes géographiques. La représentation simultanée de la nature des sols et des productions agricoles sur une carte permet de dégager les zones propices aux cultures et celles qui le sont moins. Comment décrire convenablement cette réalité sans faire appel à une représentation cartographique?

L'étude géographique d'un milieu étant essentiellement l'analyse des composantes et de leurs interrelations qui façonnent le caractère spécifique de ce milieu, on comprendra le rôle déterminant que peut

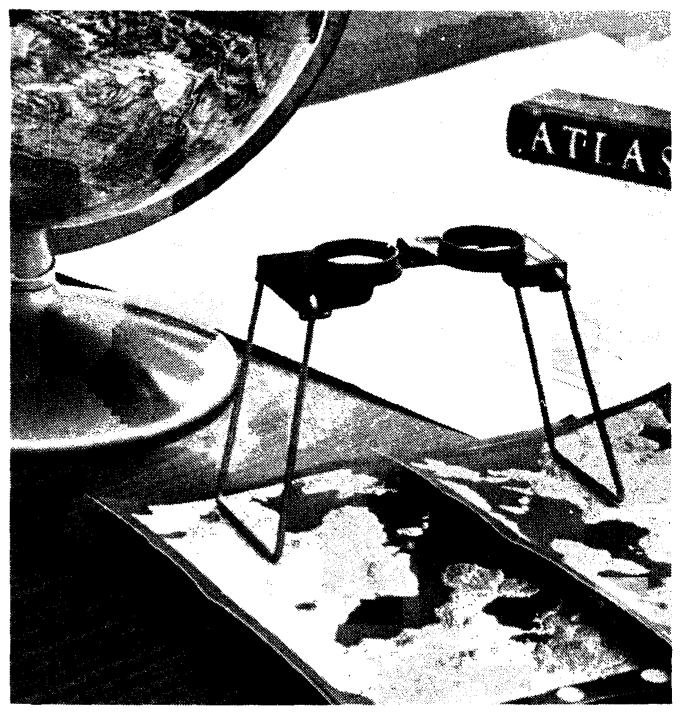

Photo Yves Tessier 
jouer la carte dans toute étude portant sur un paysage géographique, qu'il soit naturel ou humain ${ }^{1}$.

\section{Une forme privilégiée d'information sur le milieu}

Cette dimension interrelationnelle a été systématisée par le recours à l'approche écologique pour comprendre la nature profonde des phénomènes dans leurs relations avec le milieu environnant. Qu'on étudie l'oie blanche dans l'estuaire du Saint-Laurent ou le citadin dans son milieu urbain, on ne peut se passer de la carte comme on ne peut se passer de l'observation méthodique de ces phénomènes pour bien les comprendre ${ }^{2}$.

1. Par exemple la carte Canada-Énergie, établie par la Direction des levés et de la cartographie, Ministère de l'énergie, des mines et des ressources, Ottawa, 1978. 1 carte, coul., $101 \times 144 \mathrm{~cm}$ (L'atlas national du Canada, $5^{\mathrm{e}}$ éd., no MCR4002F). Ce document comprend sur une même feuille 1 carte principale à l'échelle de 1 : $7,500,000(72 \times 80 \mathrm{~cm})$ sur le pétrole et le gaz naturel, 2 cartes à l'échelle de 1:12,500,000 (42 x $44 \mathrm{~cm}$ ) sur le charbon, l'uranium et l'électricité, 1 carte à l'échelle de 1:20,000,000 $(29 \times 31 \mathrm{~cm})$ sur les débits énergétiques, 1 carte à l'échelle de $1: 25,000,000(20 \times 22 \mathrm{~cm})$ sur les degrés-jours de chauffage, 2 cartes à l'échelle de 1:35,000,000 (14 $\times 18 \mathrm{~cm}$ ) sur l'énergie solaire et l'énergie éolienne, 1 carte à l'échelle de 1:200,000,000 $(18 \times 22 \mathrm{~cm})$ sur la consommation mondiale d'énergie, 6 graphiques et 5 tableaux. Chacune des 8 cartes comporte un texte explicatif et une bibliographie sommaire. L'examen de ce document cartographique fournit des données analytiques de base et facilite l'établissement de relations entre les principales composantes liées à la situation énergétique canadienne.

2. La cartographie écologique a connu des progrès très rapides et a atteint un stade avancé de développement comme en témoigne la Carte de l'environnement et de sa dynamique [dans l'ouest de la France], publiée sous la direction de $A$. Journaux. Échelle 1:50,000. Caen, AFORMASUP, Centre de géomorphologie du C.N.R.S., 1978 cartes, coul., $66 \times 105 \mathrm{~cm}$. Le document cartographique sur la feuille imprimée représente les aspects statistiques de l'environnement: topographie, hydrographie, espaces bâtis, espaces labourés, espaces verts. Sur un calque de superposition, on a reporté les aspects dynamiques: les dégradations de la surface de la terre (naturelles ou provoquées par l'homme), la pollution de l'eau et de l'air (formes et sources de pollution), les travaux de défense et d'amélioration de l'environnement. La présentation séparée des aspects statiques et dynamiques permet d'isoler plus facilement les facteurs qui contribuent à la dégradation d'un milieu.
Outil de connaissance, la carte sert également et de plus en plus d'instrument d'action. Par exemple, si l'on fixe sur une carte les sites exceptionnels à conserver dans un paysage (chutes, points de vue, curiosités naturelles, monuments historiques, boisés remarquables) et les dangers qui les menacent (déboisement, construction de routes ou de lignes hydroélectriques, exploitation de sablières, implantation hôtelière), on peut déterminer les zones prioritaires d'intervention en vue de protéger ces sites. La conception de propositions d'aménagement repose fondamentalement sur un usage intensif de documents cartographiques d'analyse qui débouche la plupart du temps sur des cartes de communication de plans d'aménagement ${ }^{3}$.

Avec les progrès récents du langage graphique, la carte ne résulte plus uniquement du dessin de l'information mais davantage du traitement statistique et visuel de cette information et de sa représentation la plus expressive ${ }^{4}$. En effet, la carte est avant tout une image qui tend à devenir multimédia (scriptovisuelle) avec le développement récent des "one-page atlas», ces documents à caractère synthétisant qui réunissent sur une même feuille de papier des cartes proprement dites, des graphiques, des tableaux, des photos, des

3. Par exemple, la Carte de l'impact des activités humaines; Moutiers - Parc national de la Vanoise. par Pierre Gensac. Échelle 1:100,000. Grenoble, Documents de cartographie écologique, tome XIV, 1974 et Travaux scientifiques du Parc national de la Vanoise, tome VI, 1975. 1 carte, coul., $54 \times 71$ $\mathrm{cm}$. Sont reproduits les milieux susceptibles d'être modifiés par un impact agricole, urbain, industriel, touristique, ainsi que les zones protégées ou en restauration.

4. La production scientifique d'un graphique ne réside plus dans la présentation esthétique de données statistiques, mais dans le traitement significatif de l'information à l'aide d'une nouvelle science: la graphique. Voir Jacques Bertin, La graphique et le traitement graphique de l'information, Paris, Flammarion, 1977, 277 p. (Nouvelle bibliothèque scientifique). 
textes explicatifs, des bibliographies; en quelque sorte des atlas d'une page! ${ }^{5}$

\section{Un support documentaire à considérer de plus près}

Comme on le constate aisément, la carte ne peut plus être confinée au simple rôle "d'illustration» de l'information qu'on peut trouver ailleurs sous une autre forme (même si certains cartographes se cantonnent encore trop dans un simple rôle de dessinateur de cartes). La carte jouit d'une spécificité informationnelle et à ce titre elle a droit à la place qui lui revient parmi les supports de l'information, donc à une organisation valable et à une exploitation maximale dans un centre documentaire. L'utilisateur-demandeur de renseignements géographiques a droit au support qui communique le mieux ce genre d'information au même titre qu'à tous les autres supports qu'on a déjà mis à sa disposition.

De même, le futur bibliothécaire-documentaliste devrait être initié à la documentation cartographique ne serait-ce que de façon minimale, pour qu'il puisse s'occuper convenablement de ce médium scriptovisuel en expansion rapide. Les écoles de formation qui offrent des cours dans ce domaine spécialisé sont encore trop peu nombreuses. II est même étonnant que l'École de bibliothéconomie de l'Université de Montréal ait officiellement inscrit à son programme un cours dans le domaine pendant plusieurs années sans que ce cours n'ait été dispensé une seule fois!

5. La carte de l'énergie au Canada décrite dans la note 1 est un exemple récent de ces «atlas d'une page" dont la conception a été largement développée par Environnement Canada lors de la production des cartes suivantes: Great Lakes water use, échelle 1:1,584,000, 1971, no. JN359. Water use, Strait of Georgia-Puget Sound basin, échelle 1:500,000, 1973, no. ELD-2. L'axe Windsor Québec, 1974, no ELD-3. Région urbaine du détroit de Georgie, 1975, no ELD-4. Par ailleurs, une carte touristique attire l'attention par son habillage graphique soigné sans concession à la valeur scientifique du document. II s'agit de Tourist map of Livingstone and Victoria Falls, Edition 1 prepared by the British Government's Ministry of Overseas Development (Directorate of Overseas Surveys), Tolworth, England, 1970. 1 carte, coul., $83 \times 68 \mathrm{~cm}$ (D.O.S. (Misc) 572).
Par ailleurs, bon nombre de bibliothèques universitaires ont au cours des dernières années reconnu la valeur de la carte comme support d'information en créant leur propre cartothèque ou en «rapatriant» les très bonnes cartothèques déjà établies depuis des années dans les départements de géographie!

\section{La cartothèque: ses buts et objectifs et le développement de sa collection}

\section{But}

On peut formuler de la façon suivante le but d'une cartothèque située en milieu universitaire:

Fournir efficacement l'information cartographique sous toutes ses formes pour répondre aux besoins des clientèles desservies, pour des fins d'enseignement, d'apprentissage, de recherche et de perfectionnement.

Par information cartographique, on entend l'information consignée sur un document cartographique; l'information cartologique (cartologie: science de l'étude théorique et pratique des cartes) concerne l'existence du document cartographique même. Par exemple, un utilisateur peut chercher à savoir quelle est la répartition de la population de l'Italie (pour une étude démographique sur ce pays) ou demander à voir une dizaine de cartes de répartition de population (pour une étude sur les méthodes cartographiques utilisées pour représenter la répartition de la population, peu importe le pays).

L'efficacité d'une cartothèque s'exprime en termes de pertinence par rapport aux besoins à satisfaire, de rapidité dans les réponses apportées et de représentativité (exhaustivité fonctionnelle) de la production cartographique.

Les formes sont multiples: carte imprimée ou tirée sur demande, carte courante ou ancienne, carte manuscrite ou par ordinateur, atlas, globe, photographie aé- 
rienne ou par satellite, modèle en trois dimensions.

La nature des besoins varie selon le type de clientèle (professeur, étudiant, chercheur, membre du personnel non enseignant) et le but de l'utilisation de l'information cartographique: pour préparer un cours, une communication scientifique, un travail de trimestre, un voyage, une descente de rivière en canot, etc.

\section{Objectifs}

Dans la poursuite de ce but, on peut distinguer cinq secteurs d'activités qui se prêtent à la formulation d'autant d'objectifs à atteindre. Ces secteurs correspondent aux fonctions génériques de toute bibliothèque oeuvrant dans un milieu éducatif.

\section{1- DÉVELOPPEMENT}

Développer une collection cartographique apte à répondre aux besoins à satisfaire.

Activités caractéristiques: analyse et évaluation de besoins, élaboration et tenue à jour d'une politique de développement, repérage continu de la production courante par différents moyens, choix des documents conformément à la politique de développement et acquisition rapide des documents choisis.

\section{2- ORGANISATION}

Organiser l'accès à l'information cartographique en vue d'une exploitation optimale des ressources cartographiques acquises.

Activités caractéristiques: traitement intellectuel (classification, description cartobibliographique, mode de repérage) et technique (préparation matérielle, rangement, conservation) de la collection.

\section{3- COMMUNICATION}

Communiquer l'information cartographique en facilitant l'accès à la collection et en fournissant les services d'aide aux clientèles et d'information appropriés.
Activités caractéristiques: prêt, référence, assistance dans l'identification, le choix et l'utilisation des diverses sources d'information, recherche de l'information cartologique courante et rétrospective.

\section{4- FORMATION}

Former les clientèles dans l'utilisation systématique de l'information cartographique.

Activités caractéristiques: visite de la cartothèque, initiation à la recherche cartographique, introduction aux formes spécialisées de l'information cartographique, méthodologie du repérage de l'information cartographique et cartologique courante et rétrospective.

\section{5- ADMINISTRATION}

Administrer efficacement la cartothèque en vue d'atteindre les objectifs poursuivis.

Activités caractéristiques: planification, organisation, direction et évaluation des activités, recherche, participation au développement de la science et de la profession concernées.

La présente formulation des buts et objectifs d'une cartothèque universitaire reprend celle élaborée pour le Plan directeur triennal de la bibliothèque de l'Université Laval au cours de l'automne 1978.

\section{Développement d'une collection cartographique}

Le développement d'une collection cartographique pour une cartothèque universitaire peut s'effectuer selon quatre niveaux déterminés par les régions et les sujets à représenter.

\section{a) Collection de référence générale}

Cartes générales, physiques et politiques du monde, des continents et des pays les plus importants; cartes topographiques internationales du monde au $1: 1,000,000$ et au 1:2,500,000; cartes topographiques et hydrographiques à toutes les échelles du pays; atlas mondiaux généraux; photographies aérien- 
nes de la région immédiate de la cartothèque.

\section{b) Collection d'enseignement}

Cartes topographiques à moyenne échelle et cartes thématiques des régions mentionnées ci-dessus; atlas mondiaux thématiques, nationaux et historiques; photographies aériennes didactiques.

\section{c) Collection de recherche}

Pour certaines régions géographiques bien déterminées, cartes topographiques à grande échelle et cartes thématiques; atlas régionaux; cartes anciennes; toutes les cartes sur un sujet spécifique; photographies aériennes à diverses époques pour les régions étudiées en profondeur.

\section{d) Collection exhaustive}

Pour la région où se trouve la cartothèque, toutes les cartes anciennes et modernes, tous les atlas et toutes les photographies aériennes.

\section{Instruments de repérage de la production courante}

Les instruments de travail pour le repérage de la production cartographique courante sont très peu abondants et assez épars. Au niveau international, il existe une Bibliographie cartographique internationale ${ }^{6}$ annuelle qui énumère une partie de la production cartographique mondiale dont le nombre se situe entre 60,000 et 100,000 cartes par année. On peut signaler également une liste de documents facilement disponibles sur le marché, International Maps and Atlases in Print ${ }^{7}$, dont les entrées sont groupées par régions géographiques. Un grossiste-distributeur international, Geo-Center (Stuttgart), publie un catalogue très élaboré des documents cartographiques qu'il offre en vente à de nombreux

6. Bibliographie cartographique internationale, Paris, Librairie de la Faculté des sciences, 1946-

7. Kenneth Winch, International Maps and Atlases in Print, 2nd ed., London, Bowker, 1976, 866 p. pays. Pour les atlas, on doit signaler le très utile ouvrage de Gerard Alexander, Guide to Atlases $^{8}$.

Comme la très grande majorité de la production cartographique émane d'organismes gouvernementaux, il faut s'en remettre aux listes de ces producteurs (lorsqu'elles existent) pour connaître les documents disponibles ou nouvellement produits. Les listes d'acquisitions des cartothèques constituent des instruments très utiles de repérage, de même que les comptes rendus de cartes et d'atlas qui apparaissent dans diverses revues de géographie, dont la plus utile est sans contredit le Bulletin de la Geography and Map Division de la Special Libraries Association. John Wolter ${ }^{9}$ a décrit les principales sources d'information concernant les écrits sur la cartographie alors que Robert Karrow $^{10}$ a esquissé une typologie des sources d'information cartobibliographique applicable surtout aux cartes anciennes.

Pour le Québec, Pierre Lépine a compilé une liste très utile des principaux producteurs cartographiques du gouvernement. du Québec ${ }^{11}$. Par ailleurs, la Bibliographie du Québec incorporera sous peu les cartes géographiques aux documents répertoriés.

\section{Organisation de la documentation cartographique}

Dans l'effort général de normalisation de la description bibliographique, les cartothécaires ont apporté leur contribution

8. Gerard Alexander, Guide to Atlases: World, Regional, National, Thematic; an International Listing of Atlases Published since 1950, Metuchen, Scarecrow Press, 1971, 671 p. Supplement 1971-1975, 1977,373 p.

9. John Wolter, "Research tools and the literature of cartography", in The 1976 AB Bookman's Yearbook, part 1: the New and the Old; Maps and Atlases: a New World in Rare Book and Manuscript Collections, Clifton, N.J., AB Bookman Publications, 1976, p. 21-30.

10. Robert Karrow, "Cartobibliography", in The 1976 AB Bookman's Yearbook..., p. 43-52.

11. Pierre Lépine, "Producteurs cartographiques au Gouvernement du Québec», Cartologica (bulletin bimestriel d'information cartologique publié par la cartothèque de la bibliothèque de l'Université Laval), no 34 (mars 1975), 9 p. 
spécifique. Les travaux soutenus entrepris depuis une décennie par l'Association des cartothèques canadiennes et les Archives publiques du Canada ont pavé la voie à une normalisation poussée de la description cartobibliographique. Joan Winearls a résumé le déroulement de ces travaux techniques qui ont donné le ton aux autres activités similaires poursuivies à l'échelle nord-américaine et même internationale ${ }^{12}$.

\section{Normalisation cartobibliographique}

La norme internationale de description bibliographique des documents cartographiques, ISBD (CM), a été publiée en 1977 dans sa version originale anglaise et traduite en français en $1978^{13}$. Cette norme spécifique est entièrement compatible avec la norme générale $\operatorname{ISBD}(\mathrm{G})$.

Les cartothécaires canadiens ont travaillé activement à la préparation d'un manuel détaillé de catalogage des documents cartographiques. Leur expertise dans ce domaine a permis d'apporter une contribution déterminante aux travaux de révision du chapitre trois des Anglo-American Cataloguing Rules consacré aux documents cartographiques. L'harmonisation dans le temps de ces divers travaux techniques a été des plus heureuse, puisqu'elle a permis de préparer un manuel de catalogage qui respecte à la fois la norme internationale $\operatorname{SBD}(\mathrm{CM})$ et les AACR dans leur édition nouvellement révisée. Une version préliminaire de ce manuel a été étudiée lors de la réunion de novembre 1978 du Comité du catalogue collectif de l'Association des cartothèques canadiennes, la version définitive étant prévue pour la fin de 1979.

12. Joan Winearls, "Cartobibliography and map cataloging in Canada, " in The 1976 AB Bookman's Yearbook..., p. 63-70.

13. International Federation of Library Associations and Institutions, ISBD(CM): Internationa/ Standard Bibliographic Description for Cartographic Materials, London, IFLA International Office for UBC, 1977, 58 p. Traduction française établie au Département des cartes et plans de la Bibliothèque nationale de France en 1978.

\section{Format de communication}

La normalisation cartobibliographique étant maintenant assurée, l'étape principale suivante touche à l'élaboration d'un format de communication. A partir du format MARC américain pour les cartes dont la deuxième édition a été publiée en 1976, de I'UNIMARC international et du MARC canadien pour les monographies, il a été relativement facile de développer un format MARC canadien pour les cartes. La deuxième version préliminaire de ce format a été complétée en septembre 1978, grâce à une collaboration étroite entre les Archives publiques et la Bibliothèque nationale du Canada ${ }^{14}$. La version finale sera complétée au cours de l'année 1979, de même que le manuel de codification qui doit l'accompagner. L'implantation progressive du traitement automatisé des documents cartographiques pourra alors s'effectuer sans difficultés techniques particulières pour les systèmes existants de support catalographique.

\section{D'une à plusieurs entrées principales...}

Des divergences de vue ont longtemps éloigné les bibliothécaires et les cartothécaires sur la question de l'entrée principale des cartes dans la production d'un catalogue. Les uns prônaient l'entrée à l'auteur, comme le recommandait le sacro-saint code anglo-américain, les autres recommandaient et ont effectivement utilisé la région géographique, puisqu'une carte ne se repère pas principalement par son auteur, ni même par son titre, mais par sa région géographique. C'était une simple question de bon sens, à tout le moins celui de l'utilisateur, prétendaient ces derniers!

Si la Conférence de Paris de 1961 sur les principes de catalographie a statué sur le choix et la forme de l'entrée principale, l'introduction de l'automatisation a rendu ce principe assez désuet, en particulier pour les documents cartographiques, en

14. National Library of Canada. Canadian MARC Office, Canadian MARC Communication Format: Monographs, Incorporating Draft Specifications for Cartographic Materials, 2nd version, Ottawa, 1978, p.v. Circulation restreinte. 
raison de la facilité à multiplier les points d'accès au dossier bibliographique. S'il faut conserver une entrée principale à l'auteur ou au titre pour satisfaire le catalogueur scrupuleux et une entrée principale à la région pour répondre aux besoins de l'utilisateur, l'automatisation viendra héroïquement dénouer le noeud gordien, puisqu'elle permet de produire des catalogues avec l'entrée principale... au choix!

\section{Classification}

La question de la classification des documents cartographiques constitue une matière d'incidence beaucoup plus limitée que celle de la description bibliographique. Qu'il suffise de mentionner les deux systèmes les plus répandus, celui de la Library of Congress (indices G1000 à G3122 pour les atlas, G3200 à G9980 pour les cartes) ${ }^{15}$ et celui de Boggs et Lewis ${ }^{16}$, tenu à jour localement par ses utilisateurs.

Le mode de conservation le plus répandu et somme toute le plus pratique est à plat, dans des grands tiroirs peu profonds. Mary Larsgaard ${ }^{17}$ a passé en revue tous les aspects concernant la conservation physique des documents cartographiques.

\section{Exploitation reposant sur l'interface humain}

Le service de référence joue un rôle déterminant dans l'efficacité d'une bibliothèque. Ce service prend sa dimension véritable à la lumière des constatations suivantes. L'utilisateur est généralement moins familier avec l'organisation et le fonctionnement d'une cartothèque qu'il ne l'est avec celle d'une bibliothèque (quand il l'est véritablement...). II connaît la produc-

15. United States. Library of Congress, Classification, Class G: Geography, Maps, Anthropology, Recreation, 4th ed., Washington, D.C., 1976, 435 p.

16. S.W. Boggs and D. Lewis, The Classification and Cataloging of Maps and Atlases, New York, Special Libraries Association, 1945, 175 p. Traduit en français et tenu à jour à la cartothèque de la bibliothèque de l'Université Laval.

17. Mary Larsgaard, Map Librarianship, an Introduction, Littleton, Col., Libraries Unlimited, 1978, p. 155-158 (Library Science Text Series). tion cartographique moins bien que celle sous forme de livre ou de périodique. Ses besoins d'information cartographique sont plus difficiles à cerner, souvent parce qu'ils sont complémentaires de ses autres besoins documentaires. La très grande majorité des cartes émanent d'organismes gouvernementaux et sont publiées en collection, ce qui rend parfois plus complexes l'identification et le repérage de ces documents.

\section{Une gamme traditionnelle inversée}

L'expérience nous autorise à avancer les approximations suivantes concernant les moyens utilisés pour repérer la documentation cartographique dans une cartothèque: le personnel, $60 \%$; le système de classification, $25 \%$; les cartes-index, $10 \%$; le catalogue (même s'il est très bien fait et couvre l'ensemble de la documentation conservée), $5 \%$. Le premier chiffre peut surprendre, le dernier est renversant peutêtre. Ces approximations reflètent la réalité.

L'interface humain dans une cartothèque est déterminant non seulement dans le repérage de la documentation cartographique, mais également dans son utilisation. Tous les utilisateurs ne sont pas également familiers avec le langage de la communication cartographique. Ce langage évolue dans le sens d'une plus grande complexité avec les développements récents tant dans les contenus cartographiés que dans les méthodes de représentation graphique. De plus, lorsque les utilisateurs se trouvent en situation d'apprentissage à l'égard de contenus qui ne leur sont guère familiers, le travail de référence se double d'une dimension pédagogique des plus évidente et des plus utile.

\section{Le lieu vivant de l'information cartographique}

Avec l'évolution actuelle des méthodes pédagogiques vers une plus grande individualisation de l'apprentissage, une autonomie personnelle plus considérable et une diversification plus poussée des médias d'apprentissage, "l'encadrement» de la consultation documentaire devient plus 


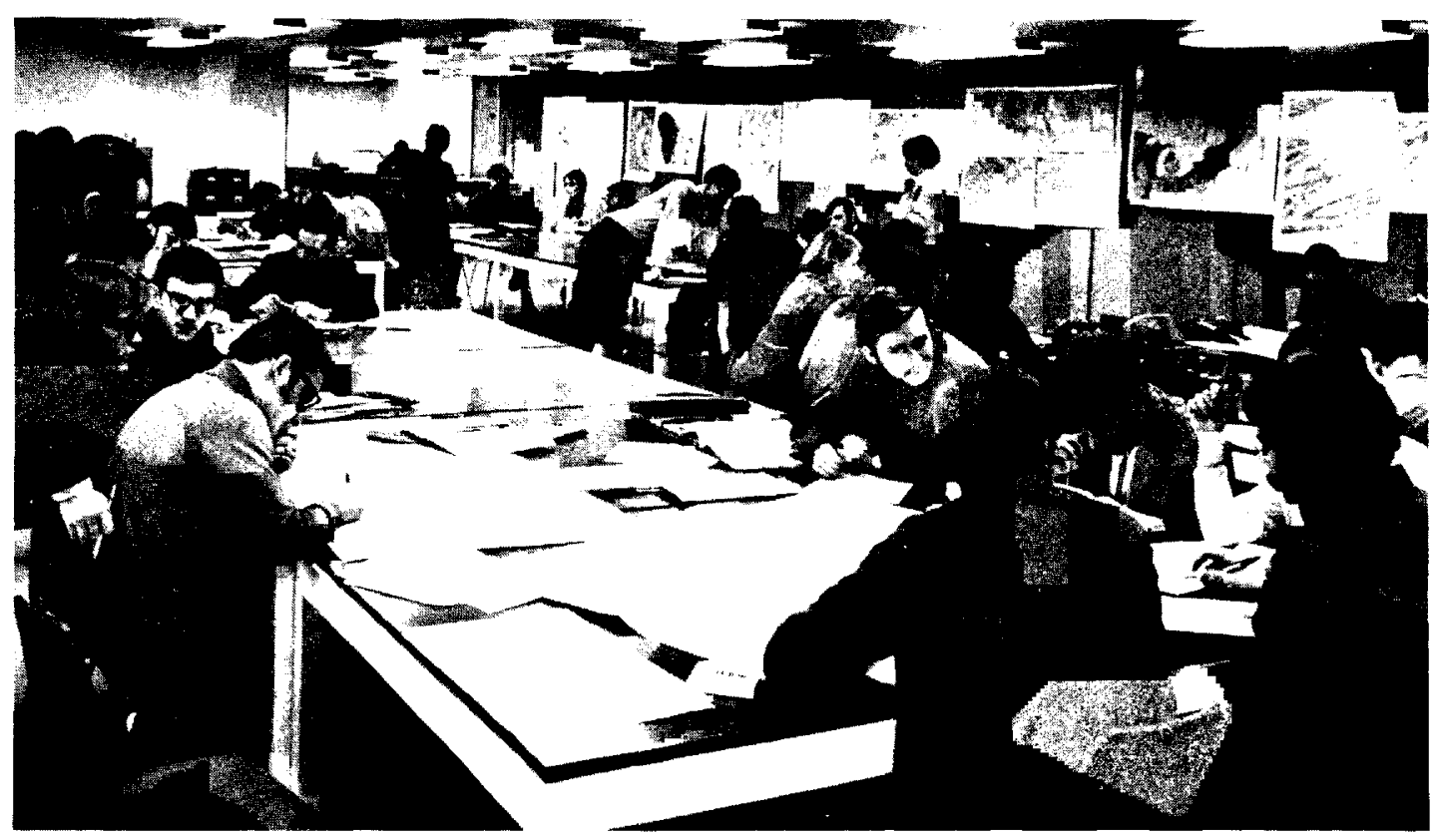

Photo Yves Tessier

exigeant pour le personnel des centres documentaires, en particulier celui des cartothèques.

Cette situation amène à multiplier les occasions de former les clientèles à l'utilisation systématique de l'information cartographique par des activités très diversifiées, comme des visites pour les nouveaux étudiants, des présentations documentaires (séminaires), des expositions, des manifestations continues destinées à faire connaître dès leur parution les nouvelles publications cartographiques, etc.

La cartothèque doit devenir le lieu vivant de l'information cartographique.

\section{De la bibliothéconomie spécialisée... à la cartothéconomie}

L'organisation plus systématique de la documentation cartographique a donné naissance à une petite communauté scientifique qui s'est développée, s'est donné un corpus de littérature sans cesse croissant et s'est constituée en groupements professionnels bien identifiés. Il est intéressant de remarquer la progression récente de ces développements.
Les dates de fondation des organismes professionnels dans ce domaine sont en elles-mêmes très significatives:

1941, États-Unis. Special Libraries Association. Geography and Map Division ${ }^{18}$;

1966, Grande-Bretagne. Map Curators' Group;

1967. Canada. Association des cartothèques canadiennes / Association of Canadian.Map Libraries $^{19}$;

1968, États-Unis (Côte ouest). Western Association of Map Libraries;

1969, International Federation of Library Associations. Geography and Map Libraries Subsection.

Ces associations ont mis sur pied plusieurs publications périodiques dont la plus utile est sans contredit le Bulletin de la

18. Special Libraries Association, Geography and Map Division, 235 Park Ave. South, New York, N.Y. 10003.

19. Association des cartothèques canadiennes/Association of Canadian Map Libraries, a/s Collection nationale de cartes et plans, Archives publiques du Canada, 395 rue Wellington, Ottawa KIA ON3. 
Geography and Map Division de la Special Libraries Association ${ }^{20}$. Elles ont également publié des répertoires de cartothèques ou de collections de cartes ${ }^{21}{ }^{22}$. Pour la France, le Laboratoire "Intergéo" de documentation et d'information géographique du C.N.R.S. publie des travaux très utiles dans le domaine de la documentation géographique et cartographique ${ }^{23}$. Au Québec, un groupe informel d'une douzaine de cartothécaires poursuit diverses activités et a déjà produit un recueil de textes sur les cartothèques universitaires du Québec ${ }^{24}$.

Comme suite logique aux développements de la profession et des connaissances spécialisées dans le domaine cartothéconomique, il était normal de voir apparaître des ouvrages tentant de résumer l'essentiel de ces connaissances et d'en faire une synthèse. Jeremiah Post a réuni huit articles dans son numéro du Drexel Library Quarterly consacré aux principaux aspects de l'organisation des cartes $^{25}$. Roman Drasniowsky a compilé

20. Bulletin of the Geography and Map Division, Special Libraries Association. Business manager, Mrs. Kathleen I. Hickey, 9927 Edward Ave., Bethesda, Maryland 20014.

21. Map Collections in the United States and Canada, 2nd ed., New York, Special Libraries Association, 1970, $159 \mathrm{p}$.

22. Lorraine Dubreuil, Directory of Canadian Map Collections/Répertoire des collections de cartes canadiennes, $3^{e}$ éd., Ottawa, Association des cartothèques canadiennes, 1977, $126 \mathrm{p}$.

23. Le Laboratoire d'information et de documentation en géographie «Intergéo», 191 rue St-Jacques, 75005 Paris publie entre autres la Bibliographie géographique internationale, la Bibliographie cartographique internationale et Intergéo bulletin. Le numéro 44 (1976) de ce bulletin comporte de précieuses indications sur la cartographie française: des bibliographies et répertoires se rapportant en tout ou en partie à la cartographie, un guide de l'édition française cartographique, quelques éléments sur la production française à la fin de 1976 et un répertoire des cartothèques de France.

24. Les cartothèques universitaires du Québec, textes édités et présentés par Yves Tessier, Québec, mars 1973, 50 p. Épuisé. Pierre Lépine a produit un document de travail très utile en janvier 1976, La cartobibliographie au Québec et la Bibliothèque nationale du Québec, $19 \mathrm{p}$.

25. Jeremiah B. Post, “Map librarianship». Drexel Library Quarterly, vol. 9, no. 4 (October 1973), $90 \mathrm{p}$. une anthologie très utile réunissant 48 articles déjà publiés et les a présentés selon une suite logique correspondant aux opérations fondamentales de l'organisation et de la mise en valeur d'une cartothè$q^{26} e^{26}$. L'auteur a produit cet ouvrage en vue d'appuyer son enseignement dans ce domaine à l'université Columbia.

L'effort de synthèse devient plus évident avec l'ouvrage de Harold Nichols qui s'attarde surtout à dégager les principes de base, plutôt que des procédures détaillées, dans l'organisation et la gestion d'une cartothèque ${ }^{27}$.

Plus récemment Mary Larsgaard a publié le manuel tant attendu par ceux qui désirent obtenir des informations simples, complètes, à jour et pratiques, lorsqu'il est question de s'occuper des cartes d'une manière systématique ${ }^{28}$.

Ce bref survol de la carte comme support de l'information scientifique et de son organisation dans les milieux documentaires a permis de constater que ce secteur a en quelque sorte développé son identité propre en moins de vingt-cinq ans.

La carte est devenue aujourd'hui un médium des plus intéressant pour la communication de l'information scientifique sous une forme scriptovisuelle. Avec l'ouvrage de Bertin sur la graphique, le manuel de Larsgaard sur la cartothéconomie et l'atlas magnifique The Earth and $\mathrm{Man}^{29}$, nous avons une trilogie cartologique de choix pour nous initier au langage de l'esprit... géographique.

26. Roman Drazniowsky, Map Librarianship: Readings, Metuchen, N.J., Scarecrow Press, 1975, $548 \mathrm{p}$.

27. Harold Nichols, Map Librarianship, London, Hamden, Conn., Clive Bingley, Linnet Books, 1976, 298 p. Voir notre compte rendu des ouvrages de Post, Drazniowsky et Nichols dans Cahiers de géographie de Québec, vol. 20, no 51 (décembre 1976), p. 572-576.

28. Mary Larsgaard, Map Librarianship..., $330 \mathrm{p}$.

29. Rand McNally and Co., The Earth and Man; a Rand McNally World Atlas, New York, 1972, $439 \mathrm{p}$. 